\title{
Factores diferenciadores en la elección de tratamiento sustitutivo renal en nuestra consulta de enfermedad renal crónica avanzada
}

\author{
Juana Heredia Fernández, Cristóbal Lorente Méndez, Felicísima Astorga Pérez, Josefa Chica Arellano
}

\section{Hospital Santa Lucia. Cartagena. Murcia}

\section{Introducción:}

El proceso de información y elección de tratamiento sustitutivo renal (TSR) en pacientes con enfermedad renal crónica avanzada (ERCA) constituye un derecho del paciente y una etapa clave para su tratamiento. Por esta motivo se puso en marcha la consulta ERCA (CERCA) en Enero 2009, con pacientes con MDRD < de 30 $\mathrm{ml} / \mathrm{min} / 1,73 \mathrm{~m} 2$. Entre los objetivos CERCA está dar información mediante documentos y consentimientos, incluyendo al paciente en un programa de formación, educación y participación activa en la evolución de su enfermedad que le permita elegir el TSR que más se adecue a sus necesidades.

Estudio retrospectivo observacional de pacientes atendidos en CERCA ( $n=197)$, desde enero 2009 y su evolución hasta diciembre 2012. Se analizan variables sociodemográficas (edad, sexo, etiología), MDRD inicial, opción de TSR inicial y final, factores de riesgo cardiovascular asociados (DM, HTA, dislipemia, tabaquismo, obesidad) e índice de Charlson.

\section{Análisis estadístico SPSS13.0.}

Las variables cuantitativas expresadas como media, desviación estándar y rango. Las variables cualitativas como frecuencia y porcentajes. Para el contraste de hipótesis ANOVA, $t$, student y chi-cuadrado (significativa estadísticamente $p<0.05$ ).

El $59.4 \%$ hombres, edad media de $67.7 \pm 13.3$, etiología más frecuente vascular / hipertensiva (37\%) con MDRD medio inicial $16.7 \pm 6.9 \mathrm{ml} / \mathrm{min} / 1.73 \mathrm{~m}^{2}$. Pacientes con elevados factores riesgo cardiovascular (FRCV):
97.5\% HTA, $77.7 \%$, DM, y $34.5 \%$ obesos con índice de masa corporal (IMC) $>30$, fumadores $44.2 \%$, dislipemicos $70.6 \%$ e I. Charlson medio $8.45 \pm 3.53$.

Existen diferencias significativas entre la elección de TSR inicial y final $(p<0.001)$.

La elección final de TSR fue: de 102 iniciales (51,8\%) de HD el 90.2\% ( $n=92)$ siguió en HD 8.8\% ( $n=9)$ TC y $0.9 \%(n=1)$ DP.

De $31(15,7 \%)$ iniciales en DP: $70.9 \%(n=22)$ siguió en DP frente al $29.1 \%(n=9)$ que optaron por HD.

De 40 iniciales en TC (20,3\%) se mantuvieron $87.5 \%$ $(n=35), 10 \%(n=4)$ decidió HD y $2.55 \%(n=1)$ DP.

No decidieron (ND) $12,2 \%(n=24)$.

Entre los factores diferenciadores de los que cambiaron de opinión no hay diferencias, salvo el MDRD ( $p=0.024)$ mayor en los que cambian, $(18.38+/$ - 8.59) versus (15.28 +/- $5.37 \mathrm{ml} / \mathrm{min} / 1.73 \mathrm{~m} 2)$.

\begin{tabular}{|c|c|c|c|c|}
\hline & HD & DP & TC & ND \\
\hline $\begin{array}{l}\text { Edad } \\
(p<0.001)\end{array}$ & $65.8+-12.7$ & $56.5+-13.5$ & $77.2+-8.9$ & $74.6+-6.5$ \\
\hline $\begin{array}{l}\text { MDRD } \\
(p<0.001)\end{array}$ & $\begin{array}{c}15.12+- \\
6.02\end{array}$ & $\begin{array}{c}15.86+- \\
5.95\end{array}$ & $\begin{array}{c}16.82+- \\
6.93\end{array}$ & $\begin{array}{c}23.75+- \\
7.87\end{array}$ \\
\hline $\begin{array}{l}\text { Charlson } \\
(p<0.001)\end{array}$ & $8.09+-3.02$ & $6.26+-3.71$ & $\begin{array}{c}10.10+- \\
3.70\end{array}$ & $\begin{array}{c}10.04+- \\
3.17\end{array}$ \\
\hline $\mathrm{N}^{0} \mathrm{FRCV}(\mathrm{ns})$ & $2.93+-1.14$ & $3.13+-1.02$ & $2.55+-1.11$ & $3.00+-0.93$ \\
\hline Sexo H (ns) & $61.8 \%$ & $67.7 \%$ & $47.5 \%$ & $58.3 \%$ \\
\hline $\begin{array}{l}\text { Etiología } \\
(p=0.016)\end{array}$ & $67.7 \%$ DM & & $\begin{array}{c}40.5 \% \\
\text { VASCU- } \\
\text { LAR }\end{array}$ & \\
\hline
\end{tabular}


Los pacientes valorados en CERCA, mayoritariamente son hombres, añosos y con alta comorbilidad y FRCV: Los pacientes que no deciden TSR tienen mayor MDRD al inicio de su valoración. Los que optan por DP, son más jóvenes y menos obesos aunque asocian más FRCV. Los que eligen TC son más añosos, con IMC elevado, mujeres y de etiología vascular/HTA (aunque sin diferencias significativas). El $13.9 \%$ de los pacientes cambian su elección inicial, fundamentalmente los que eligen DP (29.1\%). Entre los $D M$, la opción mas elegida es HD ( $(p=0.016)$. No encontramos factores diferenciables, salvo MDRD mayor en el grupo que cambia, posiblemente por el tiempo de permanencia en ERCA. Se precisa seguimiento controlado y especifico que refuerce la opción inicial del paciente.

\section{Referencias Bibliográficas}

1. Garrido López MV, Sesmero Ramos C, Portoles Perez JM. Estudio sobre el cambio de elección de tratamiento renal sustitutivo en pacientes que han optado por diálisis peritoneal. Rev. Soc. Esp. Enfer 2006 Vol. 9 n.1.

2. García Estévez $S$, Vinagre Rea G, Arribas Cobo P. Influencia de factores epidemiológicos en la elec- ción de la modalidad de tratamiento renal sustitutivo en la consulta de enfermería de ERCA. Rev. Soc Esp. Enferm Nefrol 2012 Vol. 15 n.4.

3. Lorenzo v, Consulta de enfermedad renal crónica avanzada. Experiencia de 12 años. Nefrología 2007 Vol. 27 n.4.

4. Pastor J, Julian JC. Claves del proceso de información y elección de modalidad de diálisis en pacientes con insuficiencia renal crónica. Nefrología 2007 Vol. 1. (supl 1). 20105: 15-20 n.4.

5. Sánchez Tomero JA. Planificación anticipada e inicio de diálisis. Nefrología: Vol. 29. (supl 4). 2009.

6. Bardon Otero E, Martí í Monros A, Vila Paz ML. Enfermería en la consulta de enfermedad renal crónica avanzada (ERCA). Nefrología: Vol. 28. (supl. 3): 2008: 53-56.

7. Jofré R. Factores que afectan a la calidad de vida en pacientes en prediálisis, diálisis y trasplante renal. Nefrología 1999; Vol. XIX. (supl 1): 84-90 